\title{
Convergent adaptation and ecological speciation result from unique genomic mechanisms in sympatric extremophile fishes
}

\author{
Ryan Greenway ${ }^{1 *}$, Anthony P. Brown ${ }^{2,3}$, Henry Camarillo ${ }^{1,4}$, Cassandra Delich ${ }^{1}$, Kerry L. McGowan ${ }^{2}$, \\ Joel Nelson ${ }^{2}$, Lenin Arias-Rodriguez ${ }^{5}$, Joanna L. Kelley ${ }^{2+}$, and Michael Tobler ${ }^{1 *}$ \\ ${ }^{1}$ Division of Biology, Kansas State University, Manhattan, KS, USA \\ ${ }^{2}$ School of Biological Sciences, Washington State University, Pullman, WA, USA \\ ${ }^{3}$ Current address: California National Primate Research Center, University of California, Davis, \\ Davis, CA, USA \\ ${ }^{4}$ Current address: Department of Ecology and Evolutionary Biology, Yale University, New Haven, \\ CT, USA \\ ${ }^{5}$ División Académica de Ciencias Biológicas, Universidad Juárez Autónoma de Tabasco, \\ Villahermosa, Tabasco, Mexico \\ *Corresponding authors: ryansgreenway@gmail.com; joanna.1.kelley@wsu.edu; tobler@ksu.edu
}




\title{
Significance Statement
}

Divergent lineages that coexist in sympatry and are exposed to the same sources of natural selection provide a unique opportunity to study convergent evolution across levels of organization because confounding factors associated with geographic replications are eliminated. Using three sympatric lineages of livebearing fishes inhabiting toxic and adjacent nontoxic habitats, we show that the convergent evolution of phenotypic adaptation and reproductive isolation can evolve in the absence of substantial convergence at the genomic level. Our results suggest that the nature of selection is less important in producing genomic convergence than the initial genomic substrates that are available for selection to act on.

Keywords: Adaptive evolution | Convergent evolution | Ecological genomics | Hydrogen sulfide | Local adaptation | Mitochondria | Poeciliidae | Population genomics

\begin{abstract}
Divergent selection along ecological gradients can lead to speciation, and replicated speciation events occur when populations of multiple lineages undergo divergence following colonization of similar environments. In such instances, it remains unclear to what extent reproductive isolation evolves via convergent mechanisms at the genomic level due to biases associated with shared ancestry and variation in selection associated with geographic replicates typically studied in this context. We used a unique system in which three species of poeciliid fishes occur in sympatry in a toxic, hydrogen sulfide $\left(\mathrm{H}_{2} \mathrm{~S}\right)$-rich spring and an adjacent nonsulfidic stream to examine shared patterns of adaptive divergence across multiple levels of biological organization. Despite small spatial scales, we found strong genetic differentiation between populations in sulfidic and nonsulfidic habitats mediated by strong selection against migrants between habitat types. High levels of reproductive isolation were accompanied by convergent patterns of adaptation in morphological and physiological traits, as well as genome-wide patterns of gene expression across all three species. Furthermore, the mitochondrial genomes of each species exhibit shared signatures of selection on key genes involved in $\mathrm{H}_{2} \mathrm{~S}$ toxicity. However, contrary to predictions of speciation theory, analyses of divergence across the nuclear genomes neither revealed evidence for clear genomic islands of speciation nor substantial congruence of outlier regions across population pairs. Instead, heterogenous regions of divergence spread across the genome suggest that selection for polygenic physiological adaptations likely facilitated the rapid evolution of high levels of reproductive isolation. Overall, we demonstrate that substantial convergence across multiple levels of biological organization can be mediated by nonconvergent modifications at the genomic level. By disentangling variation in natural selection from lineage-specific evolution in this system of highly divergent, yet sympatric lineages, our results emphasize the outsized role of the genomic substrate upon which selection acts in driving convergent evolution at the phenotypic level.
\end{abstract}


Reproductive isolation can evolve as a byproduct of adaptation (1). When reproductive isolation evolves in multiple lineages adapting to similar selective regimes, ecological speciation occurs convergently (1-3). Convergent evolutionary outcomes provide evidence for the repeatability and predictability of evolution (4-6), although it remains unclear under what circumstances convergence in phenotypic evolution and speciation is a consequence of the same or different underlying genetic mechanisms (7).

Convergent speciation is frequently studied in geographically disjunct but closely related population pairs exposed to divergent selection, essentially providing spatially replicated speciation events within a clade (4). The focus on closely related lineages likely biases our understanding of general patterns of convergence, because shared evolutionary responses may be the result of selection on standing genetic variation and constraints associated with shared genomic architecture (8-10). Similarly, the focus on geographically disjunct populations exposed to seemingly similar selective regimes has the potential to ignore the effects of unquantified environmental variation, which often leads to idiosyncratic evolutionary responses $(11,12)$. Comparing adaptation and speciation in distantly related taxa that are experiencing a shared selective environment in sympatry eliminates the influences of shared genetic variation and unquantified environmental factors among locations. Even though such systems contribute significantly to our understanding of the predictability and repeatability of evolution, they remain rarely studied $(13,14)$.

In the present study, we took advantage of a unique system to explore whether there are shared patterns of adaptive divergence among three sympatric lineages of livebearing fishes (Poeciliidae). In Chiapas, Mexico, populations of Poecilia mexicana, Pseudoxiphophorus bimaculatus, and Xiphophorus hellerii occur in sympatry within the La Gloria sulfide spring complex, which is characterized by naturally occurring hydrogen sulfide $\left(\mathrm{H}_{2} \mathrm{~S}\right)$, and adjacent nonsulfidic streams (Fig. 1A, B). The presence and absence of $\mathrm{H}_{2} \mathrm{~S}$ creates strong divergent selection across habitat types because of the toxic properties of $\mathrm{H}_{2} \mathrm{~S} . \mathrm{H}_{2} \mathrm{~S}$ binds to cytochrome coxidase (COX) and interrupts oxidative phosphorylation (OxPhos) in mitochondria, ultimately stopping aerobic ATP production necessary for the maintenance of cellular functions (15). At the molecular level, the clear biochemical and physiological consequences of $\mathrm{H}_{2} \mathrm{~S}$ predict adaptive modification of genes associated with OxPhos and enzymatic $\mathrm{H}_{2} \mathrm{~S}$ detoxification, which is mediated by the sulfide:quinone oxidoreductase (SQR) pathway inside of mitochondria (15).

The La Gloria system is particularly interesting because it allows for the juxtaposition of evolutionary responses to $\mathrm{H}_{2} \mathrm{~S}$ in distantly related species that occur in sympatry with evolutionary responses previously documented in geographically disparate populations of sulfide spring fishes within the family Poeciliidae [see (16) for a review]. Throughout the Americas, highly endemic poeciliids locally-adapted to sulfide springs have evolved from ancestral populations in freshwater habitats (16-18). Evolutionary responses to the toxic conditions have primarily been studied in geographically replicated populations of $P$. mexicana, which exhibit convergence in morphology, physiology, reproductive life histories, gene expression, and the concomitant emergence of 
reproductive isolation (16). While some convergence appears to result from de novo modification of the same genes or biochemical pathways (e.g., in OxPhos), there is also evidence for selection on standing genetic variation [e.g., in the SQR pathway (19-23)]. As in other systems, the focus on convergent speciation across geographically replicated lineages within P. mexicana may have biased the interpretation of the predictability of ecological speciation in sulfide springs. Indeed, a recent broad-scale study of adaptation to sulfide springs across the Poeciliidae found evidence for convergence in gene expression profiles of 10 evolutionarily independent sulfide spring lineages, but only limited evidence for repeatable patterns of molecular evolution outside of a few mitochondrially-encoded OxPhos genes (23). The degree to which this breakdown of molecular convergence at a broader phylogenetic scale is related to differences in the selective regimes among geographically disparate sulfide springs or attributable to lineage-specific evolutionary solutions to a shared source of selection remains unresolved.

The key goal of the current study was to determine whether convergent evolutionary outcomes are primarily driven by the nature of selection or by the genomic substrates that selection is acting upon. If the lack of convergence at the genomic level was a consequence of environmental variation among geographically distinct sulfide springs (23), we predicted evolutionary responses in the three sympatric lineages at La Gloria to be dominated by convergent modifications of phenotypes and underlying genes. To address this overarching hypothesis, we (i) established evidence for local adaptation and ecological speciation between sulfidic and nonsulfidic populations of each species, (ii) identified signatures of convergent evolution in phenotypic traits that have previously been associated with adaptation to sulfide spring environments (body shape, $\mathrm{H}_{2} \mathrm{~S}$ tolerance, and gene expression), and (iii) identified convergent patterns of genomic differentiation between populations. Overall, we found evidence for convergence in adaptation and speciation across multiple levels of organization, but convergence at the genomic level was limited and not associated with genomic regions predicted to respond to selection from $\mathrm{H}_{2} \mathrm{~S}$.

\section{Results and Discussion}

\section{Evidence for Local Adaptation and Ecological Speciation}

The La Gloria sulfide spring complex is spatially restricted ( $200 \mathrm{~m}$ in length) and flows directly into a nearby nonsulfidic stream (Figure $1 \mathrm{~A}$ ). There are no physical barriers that prevent fish movement between the sulfidic spring run and the nonsulfidic stream, but there are stark differences in water chemistry (Figure 1C). Besides the presence and absence of $\mathrm{H}_{2} \mathrm{~S}$, the two habitats differ in dissolved oxygen concentrations, $\mathrm{pH}$, and specific conductivity. To test for genetic signatures of reproductive isolation, we sampled 20 individuals per species in each habitat type. We conducted population genomic analyses based on whole-genome resequencing to infer contemporary population structure and historical dynamics of gene flow between the sulfidic and nonsulfidic populations. If there was local adaptation and ecological speciation, we predicted significant population structure and low rates of gene flow between habitat types. 
We used a maximum-likelihood-based assignment approach to estimate admixture proportions for all individuals using genome-wide single nucleotide polymorphisms (SNPs) (24). This approach unambiguously clustered individuals by their habitat type of origin and only detected low levels of gene flow between sulfidic and nonsulfidic populations within each species (Figure 2A). Principal components analysis corroborated these results, consistently separating populations by habitat type along the primary axis of genetic variance in each species (Figure S1). Except for two early generation hybrids in Poecilia, recent gene flow appears to be rare and occur asymmetrically from sulfidic into nonsulfidic populations. Reconstruction of demographic histories revealed differences in the timing and dynamics of divergence among population pairs (Figure S2). For each species, the best-fit demographic model supported initial divergence in isolation followed by secondary contact with gene flow between sulfidic and nonsulfidic populations. Similar patterns have been documented in other extremophile fishes in the region, and extreme habitats may serve as refugia that support relic populations while populations in adjacent normal habitats were subject to local extinction and recolonization in response to climate changes during the Pleistocene (25). Estimates of times of initial divergence and secondary contact varied substantially among the lineages present at La Gloria (8-19 ka and 1-4 ka, respectively; Figure S2), which could in part be driven by differences in the generation times of these species in the wild (our models assumed equal generation times). Demographic models also corroborated limited gene flow (less than one migrant per generation in either direction) and asymmetric movement from sulfidic into nonsulfidic populations. Overall, these results are consistent with local adaptation and ongoing ecological speciation, with greatly reduced gene flow and the maintenance of population differentiation despite small spatial scales and a lack of physical barriers between habitats.

To substantiate the findings of the population genomic analyses, we also conducted in situ reciprocal translocation experiments, which explicitly tested for local adaptation (26) and reproductive isolation through selection against migrants (27). We found strong, habitat-specific differences in the survival of sulfidic and nonsulfidic populations of each lineage (Figure $2 \mathrm{~B}$; habitat of origin $\times$ testing habitat: $P<0.001$; Table S1). Fish tested in their own habitat had significantly higher survival rates (88-100 $\%$ ) than those tested in the opposite habitat type, with the lowest survival observed for fish moved from the nonsulfidic into the sulfidic habitat (0-21\%). Fish moved from the sulfidic into the nonsulfidic habitat had comparatively high survival (68-84\%)-though significantly lower than resident nonsulfidic fish-ultimately supporting the observed asymmetry of gene flow between habitat types. This experiment confirmed local adaptation and that natural selection against migrants serves as a strong mechanism of reproductive isolation between sulfidic and nonsulfidic populations (17). While other mechanisms also contribute to reproductive isolation between sulfidic and nonsulfidic populations [e.g., behavioral isolation and biotic interactions (28-30)], local adaptation to the toxic conditions clearly plays a key role in the maintenance of reproductive isolation between populations. Given the clear evidence for convergent ecological speciation in this system, we asked whether there was evidence for convergence in the phenotypic traits that mediate adaptation to sulfide spring environments. 


\section{Evidence for Convergence in Traits Associated with Adaptation to Sulfide Springs}

Mechanisms of adaptation to $\mathrm{H}_{2} \mathrm{~S}$-rich environments have been extensively studied in poeciliid fishes, including the biochemical and physiological modifications that directly mitigate $\mathrm{H}_{2} \mathrm{~S}$ toxicity (23) and modifications of other traits that are shaped by sources of selection that are correlated with the presence of $\mathrm{H}_{2} \mathrm{~S}$ [e.g., hypoxia, increased salinity, changes in trophic resource use, competition, and predation $(16,31)]$. Here, we quantified variation in three complex traits that typically show adaptive divergence upon sulfide spring colonization: body shape, $\mathrm{H}_{2} \mathrm{~S}$ tolerance, and gene expression. We predicted that sulfide spring populations of all three focal species would exhibit convergent patterns of trait divergence in response to evolution in sulfide springs.

Previous studies have indicated that sulfide spring populations of $P$. mexicana consistently differ in body shape from adjacent populations in nonsulfidic habitats, reflecting responses to selection on oxygen acquisition in the hypoxic sulfide springs and trade-offs between fast-start and sustained swimming $(32,33)$. Consistent with previous findings, we found significant differences in body shape between sulfidic and nonsulfidic populations of all three lineages at La Gloria (habitat $X$ species: $P<0.001$, Table S2). More importantly, there was a signal of morphological convergence across the three species (habitat: $P=0.018$, Table $S 2$ ). Shared variation in body shape across habitats pertained to head size, and fish in sulfide springs exhibited larger heads than those in nonsulfidic habitats (Figure 3A). Larger head size is correlated with increased gill surface area and higher ventilation capacity in sulfide spring populations of $P$. mexicana $(32,33)$ and other fishes $(34,35)$, suggesting that convergence in body shape evolution is primarily shaped by the hypoxic conditions in sulfide springs.

Sulfide spring populations of $P$. mexicana consistently exhibit higher tolerance to acute exposures to $\mathrm{H}_{2} \mathrm{~S}$, whether it is quantified at a molecular (23) or whole-organism level (33). We measured tolerance to acute $\mathrm{H}_{2} \mathrm{~S}$ exposure in wild-caught adult fish that were first acclimated to nonsulfidic conditions in the laboratory. Survival analyses indicated that all three sulfide spring populations exhibited increases in their ability to tolerate exposure to acute $\mathrm{H}_{2} \mathrm{~S}$ relative to nonsulfidic populations (Figure 3B; habitat: $P=0.034$, Figure S3). While $\mathrm{H}_{2} \mathrm{~S}$ tolerance was clearly affected by habitat of origin, differences in tolerance between sulfidic and nonsulfidic populations also varied across the three species, with sulfidic P. mexicana having a higher $\mathrm{H}_{2} \mathrm{~S}$ tolerance than both nonsulfidic Poecilia and sulfidic populations of the other species. This observation matched microhabitat use by the three species within the sulfide spring complex; P. mexicana was the only species inhabiting immediate spring outlets with the highest $\mathrm{H}_{2} \mathrm{~S}$ concentrations, and the other two species were more common in areas with more oxygen-rich water (Figure S4).

Finally, previous studies indicated that genes associated with $\mathrm{H}_{2} \mathrm{~S}$ toxicity and detoxification exhibit convergent expression profiles among poeciliid lineages across sulfide springs $(23,36)$. We analyzed variation in genome-wide gene expression in gills from sulfidic and nonsulfidic fish. Gene expression variation exhibited a clear phylogenetic signal and clustered individuals by species and, secondarily, by habitat type (Figure 4A). The percent of differentially expressed genes between 
sulfidic and nonsulfidic populations was high but variable across species $(13.4 \%$ for Pseudoxiphophorus, $20.4 \%$ for Poecilia, and $20.9 \%$ for Xiphophorus). While most gene expression differences were species-specific (Tables S3-5), 245 genes (1.3\% of all analyzed genes; 6.3-9.8\% of differentially expressed genes in each species) exhibited convergent expression changes across all species (153 upregulated, 92 downregulated; Figure 4B, Table S6). As predicted by the biochemical and physiological effects of $\mathrm{H}_{2} \mathrm{~S}$, shared upregulated genes were significantly enriched for associations with mitochondria and biological processes involved in $\mathrm{H}_{2} \mathrm{~S}$ toxicity and detoxification, including aerobic respiration and the electron transport chain (OxPhos), enzymatic $\mathrm{H}_{2} \mathrm{~S}$ detoxification, and the processing and transport of sulfur compounds (Table S7-10). Of particular interest, we found consistent upregulation of genes associated with the primary target of $\mathrm{H}_{2} \mathrm{~S}$ toxicity (COX) and the primary $\mathrm{H}_{2} \mathrm{~S}$ detoxification pathway (SQR pathway; Figure 4C-E). In some sulfidic populations of $P$. mexicana, notably less divergent from their nonsulfidic counterparts than populations examined in this study, expression variation in $\mathrm{H}_{2} \mathrm{~S}$-processing and OxPhos-related genes has a heritable basis (37). Ultimately, shared expression patterns across the three sulfide spring populations provide robust support for convergent regulation of genes with known roles in $\mathrm{H}_{2} \mathrm{~S}$ toxicity and detoxification.

Convergence in the expression of genes related to $\mathrm{H}_{2} \mathrm{~S}$ toxicity and detoxification in the three sympatric lineages investigated here was not substantially different from patterns observed in geographically replicated sulfidic populations of $P$. mexicana (36) or in sulfide spring lineages that span multiple genera spread across the Americas (23). This finding suggests that neither evolutionary divergence among replicated sulfide spring lineages nor variation in the selective environments experienced among lineages greatly influenced the degree of convergence. Rather, physiological constraints in responding to $\mathrm{H}_{2} \mathrm{~S}$ toxicity likely drives the repeatable changes in the regulation of predictable genes and pathways among divergent lineages and across multiple sulfide springs. Considering the evidence for convergent phenotypic evolution, and particularly the repeated modulation of genes associated with $\mathrm{H}_{2} \mathrm{~S}$ toxicity and detoxification, is there evidence for convergence at the genomic level?

\section{Evidence for Convergence at the Genomic Level}

The explicit biochemical and physiological effects of $\mathrm{H}_{2} \mathrm{~S}$ - along with the convergent changes at the transcriptome level-predict clear targets of selection at the genomic level. We first explored potential convergent changes in the mitochondrial genome, which includes genes for three subunits of $\mathrm{H}_{2} \mathrm{~S}^{\prime}$ primary toxicity target (COX). Mitochondrial haplotype networks revealed that sulfidic populations harbor mitochondrial lineages that are highly divergent from those in the nonsulfidic populations (Figure 5A). Except for one apparent first-generation hybrid in $P$. mexicana, nonsulfidic mitochondrial haplotypes were not found in sulfidic populations, suggesting a critical role of mitochondrial DNA in mediating adaptation to the toxic conditions. Consistent with the asymmetric patterns of gene flow, we also found sulfidic mitochondrial haplotypes in nonsulfidic habitats, especially in $P$. mexicana and $X$. hellerii. Using analyses of molecular evolution, we detected evidence for positive selection (elevated nonsynonymous to synonymous substitution rates, $\omega$ ) acting on five 
mitochondrial genes in the three sulfidic populations, including two COX subunits (COX1 and COX3) and genes encoding subunits of OxPhos complexes I (ND2 and ND3) and III (CYTB; Table S11), corroborating previous analyses of sulfide spring fishes $(19,23)$.

Unlike in the mitochondrial genome, recombination in the nuclear genome can mediate highly heterogeneous divergence during ecological speciation, with genomic regions directly involved in adaptation exhibiting disproportionally high divergence (genomic islands of divergence) against a background of regions of relatively low divergence that are homogenized by gene flow $(38,39)$. We predicted convergent genomic islands of divergence between sulfidic and nonsulfidic populations across the three species, including regions with mitochondrially-functioning genes that are involved in $\mathrm{H}_{2} \mathrm{~S}$ toxicity and detoxification. Considering the evidence for selection on mitochondrially encoded genes, we particularly expected to detect evidence for mito-nuclear coevolution (40), with disproportionally high divergence in the nuclear subunits of OxPhos complexes I, III, and IV. To determine if selection on the same genomic regions underlies convergent adaptation to sulfide spring environments, we used the whole-genome resequencing data described above to characterize the landscape of genomic divergence in each population pair using a combination of relative $\left(F_{\mathrm{ST}}\right)$ and absolute $\left(d_{\mathrm{XY}}\right)$ divergence metrics in non-overlapping 25 - $\mathrm{kb}$ windows $(41,42)$. Global estimates of relative divergence roughly scaled with divergence times between the sulfidic and nonsulfidic populations of each species (mean $F_{\mathrm{ST}}=0.23$ for Poecilia, 0.06 for Pseudoxiphophorus, and 0.03 for Xiphophorus; Figure S5), while absolute divergence across the genome was similar (mean $d_{\mathrm{XY}}=0.003$ for Poecilia, 0.004 for Pseudoxiphophorus, and 0.003 for Xiphophorus; Figure S6). Rather than finding clear genomic islands of divergence and convergence among species pairs, we observed heterogeneous divergence between sulfidic and nonsulfidic populations of each species across much of the genome.

Using a composite outlier analysis that simultaneously considered relative and absolute divergence (43), we found little evidence for convergent responses to selection across the three population pairs (Figure 5B). Only eight outlier regions, containing 24 genes, were shared across all three species, and 61-95 outliers were shared between pairs of the three species (Table S12). More so, we found no evidence that genes typically implicated in $\mathrm{H}_{2} \mathrm{~S}$ adaptation were associated with shared or even species-specific outlier regions, including genes of the SQR pathway that are involved in enzymatic $\mathrm{H}_{2} \mathrm{~S}$ detoxification or nuclear genes associated with COX and other OxPhos components.

Adaptation to $\mathrm{H}_{2} \mathrm{~S}$ may therefore primarily be driven by changes in the regulation of detoxification genes, with different mechanisms involved in producing the convergent gene expression patterns uncovered by transcriptome analyses. Accordingly, it is possible that the detected outlier regions harbor regulatory elements mediating adaptive shifts in gene expression (44), and divergent selection acting on regulatory elements has been implicated in adaptive divergence for several well-known models of ecological speciation (45-47). Furthermore, the evolution of an $\mathrm{H}_{2} \mathrm{~S}$-resistant COX (19, 23) may solely be driven by modification of mitochondrial subunits that form the reactive center of the protein and without coevolutionary changes in nuclear subunits. While we have evidence for 
selection on mitochondrial genes (48), it remains unclear how genetic variation in mitochondrial and nuclear loci contribute to adaptive variation in OxPhos.

Despite the lack of evidence for selection on genes associated with $\mathrm{H}_{2} \mathrm{~S}$ detoxification and OxPhos, functional annotation of shared and unique outlier regions did find evidence for enrichment of genes involved in oxygen transport and immune function (Tables S13-18). Evidence for selection on oxygen transport proteins, such as hemoglobin, is not surprising, as $\mathrm{H}_{2} \mathrm{~S}$ is capable of binding to and impairing hemeprotein function $(49,50)$. The presence of hemoglobin genes in outlier regions also confirms findings of positive selection on hemoglobin genes in other sulfide spring poeciliids (51). In contrast, the large number of genes associated with immune function was not expected, although immune genes are known hotspots for selection in vertebrates (52). Selection on immune genes could be driven by differences in parasite and pathogen communities between sulfidic and nonsulfidic habitats, which have been documented in another sulfide spring inhabited by a poeciliid fish (53). Alternatively, there have been long-standing, but poorly investigated, hypotheses about the potential role of $\mathrm{H}_{2} \mathrm{~S}$-oxidizing microsymbionts in adaptation to sulfide spring environments (15, 54), and changes in host-microbe interactions upon sulfide spring colonization may also exert selection on immune genes (55).

Considering the unexpected results, we also interrogated the genomic data through site-frequencyspectrum based scans for selective sweeps (56) in each sulfidic population and largely found similar results. No selective sweep outlier regions were shared among all three species (Figure S7), and the small subset of genes shared among pairs of species was not enriched for any biological functions (Table S19). Pseudoxiphophorus bimaculatus exhibited enrichment of both ion transport and oxygen transport genes, as well as evidence for a selective sweep in the region containing $S Q R$, a key $\mathrm{H}_{2} \mathrm{~S}$ detoxification enzyme (Table S20). However, neither P. mexicana nor X. hellerii exhibited any evidence for selective sweeps in regions containing genes related to sulfidic conditions (Table S21).

\section{Conclusions}

The three poeciliid fishes that have colonized the La Gloria sulfide spring complex provided a unique opportunity to examine how distantly related lineages respond to the same sources of divergent selection, shedding light into what aspects of evolutionary responses are repeatable and predictable. We found strong evidence for local adaptation and ecological speciation in each of the lineages and detected remarkable signatures of convergence in phenotypic traits that span levels of organismal organization, the emergence of reproductive isolation, and selection on mitochondriallyencoded genes associated with $\mathrm{H}_{2} \mathrm{~S}$ toxicity. However, evidence for convergence in the nuclear genomes was scant and not associated with genomic regions predicted to respond to selection from $\mathrm{H}_{2} \mathrm{~S}$. So, the landscapes of genomic divergence are highly idiosyncratic even when species experience the same selective regime in sympatry, suggesting that evolutionary outcomes are contingent on the genomic substrates that selection is acting on. 
Even though $\mathrm{H}_{2} \mathrm{~S}$ has clear-cut biochemical and physiological effects, tolerance to this toxicant is a polygenic trait. OxPhos complexes alone contain over 80 nuclear-encoded genes (57), and dozens of genes contribute to the physiological processing of $\mathrm{H}_{2} \mathrm{~S}$ and other sulfur compounds $(58,59)$. More importantly, environmental gradients between sulfidic and adjacent nonsulfidic habitats are complex, and the multifarious sources of selection have caused adaptive modifications of whole suites of complex traits beyond $\mathrm{H}_{2} \mathrm{~S}$ tolerance, and most traits likely have different genomic bases (16). The polygenetic nature of adaptation to sulfide spring environments ultimately reduces the likelihood of convergence at the genomic level and complicates the detection of loci underlying adaptation in specific lineages. Redundancy in the genetic basis underlying polygenic adaptation can cause lineagespecific responses to selection that are in part shaped by historical contingencies $(60,61)$. Hence, modifications at different loci can have equivalent functional consequences, such that genomic signatures that arise in response to a shared source of selection are unique to a specific lineage even when there is strong evidence for convergence in emerging traits (62). Even when convergence is not expected at the genomic level, just detecting potential genomic regions involved in adaptation can be challenging for polygenic traits, because allele frequency shifts at relevant loci can be transient or too subtle to be detectable by outlier approaches $(63,64)$. This problem can be exacerbated when adaptive evolution gives rise to reproductive isolation. Selection on small-effect loci spread across the genome can facilitate strong and stable reductions in gene flow over short timescales $(65,66)$, as observed for all three lineages in our study. High levels of background divergence resulting from post-speciation divergence can further obscure the signatures of selection on the loci mediating adaptation and reproductive isolation during earlier stages of speciation (67-69).

Despite ambiguity in the specific mechanisms of adaptive divergence at the genomic level, our results clearly document the rapid evolution of genome-wide divergence and strong reproductive isolation between sulfidic and nonsulfidic populations in three highly divergent lineages. In many ways, the limitations of our study are emblematic for our understanding of physiological adaptation in many natural systems, both in terms of the detection of genomic regions underlying polygenic adaptation and the documentation of repeatable and predictable genomic signatures in response to a shared selective regime. There has been substantial progress in understanding the molecular basis of adaptive trait evolution, and in some instances, clear evidence for convergent genomic signatures of adaptation when selection does not act on shared pools of standing genetic variation and traits are complex $(70,71)$. But the reality is that much of our understanding of the genetics of adaptation and speciation comes from systems with relatively simple traits governed by large-effect alleles. Future research elucidating the genomic underpinnings of physiological adaptation and concomitant speciation consequently needs to focus on closing gaps in our understanding of how variation in genotypes relates to the expression of traits mediating adaptation and reproductive isolation. We now know that convergence at higher levels of biological organization can be caused by nonconvergent modifications at the genomic level. The remaining question is how exactly that happens. Answering it will require better analytical integration of datasets that link processes across levels of organismal organization-from genetic variation that impacts the function of proteins, the 
regulation of genes, and ultimately the expression of relevant functional traits—and across phylogenetically replicated lineages subject to similar sources of selection.

\section{Methods}

The following sections provide a synopsis of the procedures used in this study. Detailed materials and methods are provided in the SI Appendix.

\section{Sampling}

All samples of P. mexicana, P. bimaculatus, and X. hellerï were collected from the La Gloria spring complex (sulfidic habitat) and the adjacent Arroyo Caracol (nonsulfidic habitat) in Chiapas, Mexico. These habitats are part of the Río Pichucalco drainage and located near the city of Teapa, Tabasco (see Table S22 for details).

\section{Population Structure and Demographic Modeling}

We resequenced the genomes of 20 individuals per population using an Illumina HiSeq 2500 with paired-end, 100-bp chemistry. Reads were mapped to the Xiphophorus maculatus reference genome (72), and the Genome Analysis Toolkit (73) was used to identify single nucleotide polymorphisms (SNPs). To assess population structure within each of the population pairs, we produced a set of unlinked SNPs for each species and used a maximum-likelihood-based assignment method that estimates admixture proportions for each individual (24). We also used diffusion-approximations for demographic inference $(\partial a \partial \imath)(74)$ to reconstruct the demographic history for each of the population pairs.

\section{Translocation Experiment}

We conducted reciprocal translocation experiments in 20-L mesocosms placed directly in sulfidic and nonsulfidic stream reaches $(17,75)$. Adult fish were collected by seine, and five haphazardly chosen individuals of the same species and habitat type were introduced into a mesocosm. Half of the mesocosms in each habitat were used as controls to test the survival of resident fish, and the other half to test survival of fish from the opposite habitat type. Experiments ran for 20 hours before quantification of survival rates. To analyze variation in survival, we used a generalized linear mixed model (GLMM) with a binomial error distribution and a logit-link function, including species (Poecilia, Pseudoxiphophorus, or Xiphophorus), habitat of origin (sulfidic vs. nonsulfidic), and testing habitat (sulfidic vs. nonsulfidic) as fixed effects, standard length ( $\log _{10}$-transformed) as a covariate, and mesocosm ID as a random factor.

\section{Body Shape Analysis}

Body shape analyses was based on lateral photographs on which we digitized 14 morphological landmarks (Figure S8) for geometric morphometric analyses (76). Landmark coordinates were aligned via least-squares superimposition and then analyzed using a Procrustes ANOVA (77). Predictor variables included species, habitat of origin, and sex as factors, as well as centroid size as a covariate. 


\section{$\mathrm{H}_{2} \mathrm{~S}$ Tolerance}

We quantified $\mathrm{H}_{2} \mathrm{~S}$ tolerance of adult wild-caught fish acclimated to laboratory conditions by exposing them to rapidly increasing concentrations of $\mathrm{H}_{2} \mathrm{~S}$ (33). Individual fish were placed into clear plastic containers with $150 \mathrm{~mL}$ water from the holding tanks and allowed to acclimate for 5 minutes. Following acclimation, $10 \mathrm{~mL}$ of $\mathrm{H}_{2} \mathrm{~S}$ solution were added to the experimental container at 2-minute intervals. We measured the time until the fish lost equilibrium, at which point the fish was removed from the container and placed into a recovery tank. Experiments were ended after 32 minutes (15 sulfide additions) if a fish did not lose equilibrium. We analyzed variation in $\mathrm{H}_{2} \mathrm{~S}$ tolerance using a survival analysis (Cox regression) (78). We used time to loss of equilibrium as the response variable, and individuals that did not lose equilibrium during the experiment were censored. Body mass ( $\log _{10}$ transformed), species, sex, and habitat of origin (sulfidic vs. nonsulfidic) were used as predictor variables.

\section{Comparative Transcriptomics}

To quantify genome-wide gene expression patterns, we collected gill tissues in the field and preserved them in RNAlater. mRNA was isolated from gill tissues, and cDNA libraries were constructed for each sample as described for samples in previous studies $(23,36)$. cDNA libraries were sequenced on an Illumina HiSeq 2500 with paired-end, 100-bp reads. After trimming, raw RNAseq reads were mapped to the $X$. maculatus reference genome, and we used STRINGTIE (79, 80) to quantify the number of reads that mapped to each gene. We then removed genes that did not have at least two counts per million in 3 or more individuals across all species, resulting in a set of 18,598 genes for analysis of gene expression patterns. To identify differentially expressed genes between sulfidic and nonsulfidic populations of each species, we used generalized linear models (GLMs) as implemented in the Bioconductor package EDGER (81). Finally, we used Gene Ontology (GO) enrichment analyses (82) to explore the putative biological functions of differentially expressed genes.

\section{Mitochondrial Genome Assembly and Molecular Evolution}

We assembled mitochondrial genomes from raw whole-genome resequencing reads using NOVOPlasty (83) and identified the sequences for the 13 protein-coding genes using

MitoAnnotator (84). We then used PopART (85) to calculate a TCS haplotype network and visualize variation in mitochondrial genomes of each species. We also analyzed patterns of molecular evolution in mitochondrially encoded genes to test for positive selection in lineages from sulfidic habitats. We first generated a consensus sequence for each gene and population and then used branch models implemented in the program codeml from the PAML package to test for elevated nonsynonymous to synonymous substitution rates (86).

\section{Characterizing the Genomic Landscape of Divergence}

To characterize the landscape of genomic divergence, we calculated genomic differentiation $\left(F_{\mathrm{ST}}\right.$, relative divergence) and divergence ( $d_{\mathrm{XY}}$, absolute divergence) between the sulfidic and nonsulfidic 
population of each species across non-overlapping $25-\mathrm{kb}$ windows spanning the genome. To identify probable targets of divergent selection between sulfidic and nonsulfidic populations, we used a composite outlier analysis of both statistics as implemented in the $\mathrm{R}$ package MINOTAUR (42). First, we converted $F_{\mathrm{ST}}$ and $d_{\mathrm{XY}}$ to rank-based $P$-values using a right-tailed test, then $-\log _{10^{-}}$ transformed these $P$-values, and calculated the Mahalanobis distances among windows (43). We considered windows in the top $1 \%$ of the resulting Mahalanobis distance distribution as genomic regions likely experiencing divergent natural selection between habitat types in each species. To complement outlier analyses, we also implemented the cross-population composite likelihood ratio [XP-CLR (56)] test to identify regions of the genome with evidence for selective sweeps in the sulfide spring population of each species based on differentiation in multilocus allele frequencies compared to the nonsulfidic population. As for gene expression analyses, we used Gene Ontology (GO) enrichment analyses (82) to explore the putative biological functions of genes that are putatively under selection.

\section{Data Availability}

All sequence data are available at the National Center for Biotechnology Information (NCBI) (https://www.ncbi.nlm.nih.gov under BioProject) (accession no. PRJNA738871).

\section{Acknowledgements}

We are indebted to the community of Teapa, especially the owners of Rancho La Gloria, for providing access to study sites, and to the Centro de Investigación e Innovación para la Enseñanza y Aprendizaje (CIIEA) for their hospitality and support during the nearly decade-long course of this project. We thank N. Barts, C. Carson, Z. Culumber, T. Doumas, G.W. Hopper, C.N. Passow, and E.A. Renner for assistance in the field, as well as O. Cornejo, K. B. Gido, A. G. Hope, D. A. Marques, and T.J. Morgan for their comments and discussions. This work was supported by grants from the NSF (IOS-1463720, IOS-1557795, IOS-1557860, and IOS-1931657) and the US Army Research Office (W911NF-15-1-0175, W911NF-16-1-0225) to JLK. and MT. RG was supported by the American Museum of Natural History (Theodore Roosevelt Memorial Fund grant), Friends of Sunset Zoo (Conservation Scholar Program grant), American Livebearer Association (Vern Parish Fund award), Society for the Study of Evolution (Rosemary Grant Advanced Graduate Research Excellence Grant), American Society of Naturalists (Student Research Award), and a NSF Graduate Research Fellowship (DGE-1746899).

\section{References}

1. P. Nosil, Ecological Speciation (Oxford University press, 2012).

2. H. D. Rundle, L. Nagel, J. Wenrick Boughman, D. Schluter, Natural selection and parallel speciation in sympatric sticklebacks. Science 287, 306-308 (2000).

3. E. B. Rosenblum, L. J. Harmon, "Same but different": replicated ecological speciation at White Sands. Evolution 65, 946-960 (2011). 
4. D. Schluter, L. M. Nagel, Parallel speciation by natural selection. Am. Nat. 146, 292-301 (1995).

5. R. Kaeuffer, C. L. Peichel, D. I. Bolnick, A. P. Hendry, Parallel and nonparallel aspects of ecological, phenotypic, and genetic divergence across replicate population pairs of lake and stream stickleback. Evolution 66, 402-418 (2012).

6. Z. D. Blount, R. E. Lenski, J. B. Losos, Contingency and determinism in evolution: Replaying life's tape. Science 362, eaam5979 (2018).

7. E. B. Rosenblum, C. E. Parent, E. E. Brandt, The molecular basis of convergent evolution. Annu. Rev. Ecol. Evol. Syst. 45, 203-226 (2014).

8. G. L. Conte, M. E. Arnegard, C. L. Peichel, D. Schluter, The probability of genetic parallelism and convergence in natural populations. Proceedings of the Royal Society B 279, 5039-5047 (2012).

9. P. F. Colosimo, et al., Widespread parallel evolution in sticklebacks by repeated fixation of ectodysplasin alleles. Science 307, 1928-1933 (2005).

10. V. Soria-Carrasco, et al., Stick insect genomes reveal natural selection's role in parallel speciation. Science 344, 738-742 (2014).

11. Y. E. Stuart, et al., Contrasting effects of envrionment and genetics generate a continuum of parallel evolution. Nature Ecology \& Evolution 1, 0158 (2017).

12. H. E. Morales, et al., Genomic architecture of parallel ecological divergence: Beyond a single environmental contrast. Sci. $A d v$. 5, eaav9963 (2019).

13. E. B. Rosenblum, L. J. Harmon, "Same same but different": replicated ecological speciation at White Sands. Evolution 65, 946-960 (2011).

14. J. A. M. Raeymaekers, et al., Adaptive and non-adaptive divergence in a common landscape. Nat. Commun. 8 (2017).

15. M. Tobler, C. N. Passow, R. Greenway, J. L. Kelley, J. H. Shaw, The evolutionary ecology of animals inhabiting hydrogen sulfide-rich environments. Annu. Rev. Ecol. Evol. Syst. 47, 239-262 (2016).

16. M. Tobler, J. L. Kelley, M. Plath, R. Riesch, Extreme environments and the origins of biodiversity: Adaptation and speciation in sulphide spring fishes. Mol. Ecol. 27, 843-859 (2018).

17. M. Plath, et al., Genetic differentiation and selection against migrants in evolutionarily replicated extreme environments. Evolution 67, 2647-2661 (2013).

18. R. Riesch, et al., Extremophile Poeciliidae: multivariate insights into the complexity of speciation along replicated ecological gradients. BMC Evol. Biol. 16, 136 (2016). 
19. M. Pfenninger, et al., Parallel evolution of cox genes in H2S-tolerant fish as key adaptation to a toxic envrionment. Nat. Commun. 5, 3873 (2014).

20. M. Pfenninger, et al., Unique evolutionary trajectories in repeated adaptation to hydrogen sulphide-toxic habitats of a neotropical fish (Poecilia mexicana). Mol. Ecol. 24, 5446-5459 (2015).

21. A. P. Brown, et al., Local ancestry analysis reveals genomic convergence in extremophile fishes. Philos. Trans. R. Soc. Lond. B Biol. Sci. 374, 20180240 (2019).

22. A. P. Brown, L. Arias-Rodriguez, M.-C. Yee, M. Tobler, J. L. Kelley, Concordant Changes in Gene Expression and Nucleotides Underlie Independent Adaptation to Hydrogen-Sulfide-Rich Environments. Genome Biol. Evol. 10, 2867-2881 (2018).

23. R. Greenway, et al., Convergent evolution of conserved mitochondrial pathways underlies repeated adaptation to extreme environments. Proc. Natl. Acad. Sci. U. S. A. 117, 16424-16430 (2020).

24. A. Raj, M. Stephens, J. K. Pritchard, fastSTRUCTURE: variational inference of population structure in large SNP data sets. Genetics 197, 573-589 (2014).

25. U. Strecker, V. H. Faundez, H. Wilkens, Phylogeography of surface and cave Astyanax (Teleostei) from Central and North America based on cytochrome b sequence data. Mol. Phylogenet. Evol. 33, 469-481 (2004).

26. T. J. Kawecki, D. Ebert, Conceptual issues in local adaptation. Ecol. Iett. 7, 1225-1241 (2004).

27. P. Nosil, T. H. Vines, D. J. Funk, Perspective: Reproductive isolation caused by natural selection against immigrants from divergent habitats. Evolution 59, 705-719 (2005).

28. R. Greenway, S. Drexler, L. Arias-Rodriguez, M. Tobler, Adaptive, but not conditiondependent, body shape differences contribute to assortative mating preferences during ecological speciation. Evolution 70, 2809-2822 (2016).

29. D. Bierbach, et al., Predator avoidance in extremophile fish. Life 3, 161-180 (2013).

30. D. Bierbach, L. Arias-Rodriguez, M. Plath, Intrasexual competition enhances reproductive isolation between locally adapted populations. Curr. Zool. 64, 125-133 (2018).

31. M. Tobler, M. Plath, "Living in extreme habitats" in Ecology and Evolution of Poecilizd Fishes, J. Evans, A. Pilastro, I. Schlupp, Eds. (University of Chicago Press, 2011), pp. 120-127. 
32. H. Camarillo, L. Arias Rodriguez, M. Tobler, Functional consequences of phenotypic variation between locally adapted populations: Swimming performance and ventilation in extremophile fish. J. Evol. Biol. 33, 512-523 (2020).

33. M. Tobler, et al., Evolution in extreme environments: replicated phenotypic differentiation in livebearing fish inhabiting sulfidic springs. Evolution 65, 2213-2228 (2011).

34. R. B. Langerhans, L. J. Chapman, T. J. DeWitt, Complex phenotype-environment associations revealed in an East African cyprinid. J. Evol. Biol. 20, 1171-1181 (2007).

35. L. J. Chapman, F. Galis, J. Shinn, Phenotypic plasticity and the possible role of genetic assimilation: Hypoxia-induced trade-offs in the morphological traits of an African cichlid. Ecol. Lett. 3, 387-393 (2000).

36. J. L. Kelley, et al., Mechanisms underlying adaptation to life in hydrogen sulfide-rich environments. Mol. Biol. Evol. 33, 1419-1434 (2016).

37. C. N. Passow, et al., The roles of plasticity and evolutionary change in shaping gene expression variation in natural populations of extremophile fish. Mol. Ecol. 26, 6384-6399 (2017).

38. M. Ravinet, et al., Interpreting the genomic landscape of speciation: a road map for finding barriers to gene flow. J. Evol. Biol. 30, 1450-1477 (2017).

39. P. Nosil, J. L. Feder, Genomic divergence during speciation: causes and consequences. Philos. Trans. R. Soc. Lond. B Biol. Si. 367, 332-342 (2012).

40. D. M. Rand, R. A. Haney, A. J. Fry, Cytonuclear coevolution: the genomics of cooperation. Trends Ecol. Evol. 19, 645-653 (2004).

41. T. E. Cruickshank, M. W. Hahn, Reanalysis suggests that genomic islands of speciation are due to reduced diversity, not reduced gene flow. Mol. Ecol. 23, 3133-3157 (2014).

42. R. Verity, et al., minotaur: A platform for the analysis and visualization of multivariate results from genome scans with R Shiny. Mol. Ecol. Resour. 17, 33-43 (2017).

43. K. E. Lotterhos, et al., Composite measures of selection can improve the signal-to-noise ratio in genome scans. Methods Ecol. Evol. 8, 717-727 (2017).

44. K. L. Mack, M. W. Nachman, Gene Regulation and Speciation. Trends Genet. 33, 68-80 (2017).

45. F. O. Hebert, S. Renaut, L. Bernatchez, Targeted sequence capture and resequencing implies a predominant role of regulatory regions in the divergence of a sympatric lake whitefish species pair (Coregonus clupeaformis). Mol. Ecol. 22, 4896-4914 (2013). 
46. O. Seehausen, et al., Genomics and the origin of species. Nat. Rev. Genet. 15, 176-192 (2014).

47. A. Ishikawa, et al., Different contributions of local- and distant-regulatory changes to transcriptome divergence between stickleback ecotypes. Evolution 71, 565-581 (2017).

48. G. E. Hill, Mitonuclear Ecology. Mol. Biol. Evol. 32, 1917-1927 (2019).

49. R. Pietri, E. Roman-Morales, J. Lopez-Garriga, Hydrogen sulfide and heme proteins: knowledge and mysteries. Antioxid. Redox Signal. 15, 393-404 (2011).

50. S. V. Evans, B. P. Sishta, A. G. Mauk, G. D. Brayer, Three-dimensional structure of cyanometsulfmyoglobin C. Proc. Natl. Acad. Sci. U. S. A. 91, 4723-4726 (1994).

51. N. Barts, et al., Molecular evolution and expression of oxygen transport genes in livebearing fishes (Poeciliidae) from hydrogen sulfide rich springs. Genome 61, 273-286 (2018).

52. A. J. Shultz, T. B. Sackton, Immune genes are hotspots of shared positive selection across birds and mammals. Elife 8 (2019).

53. M. Tobler, et al., Selection from parasites favours immunogenetic diversity but not divergence among locally adapted host populations. J. Evol. Biol. 27, 960-974 (2014).

54. S. Hotaling, et al., Bacterial Diversity in Replicated Hydrogen Sulfide-Rich Streams. Microb. Ecol. 77, 559-573 (2019).

55. L. V. Hooper, D. R. Littman, A. J. Macpherson, Interactions between the microbiota and the immune system. Science 336, 1268-1273 (2012).

56. H. Chen, N. Patterson, D. Reich, Population differentiation as a test for selective sweeps. Genome Res. 20, 393-402 (2010).

57. F. Zhang, R. E. Broughton, Mitochondrial-nuclear interactions: compensatory evolution or variable functional constraint among vertebrate oxidative phosphorylation genes? Genome Biol. Evol. 5, 1781-1791 (2013).

58. K. R. Olson, H2S and polysulfide metabolism: Conventional and unconventional pathways. Biochem. Pharmacol. 149, 77-90 (2018).

59. M. H. Stipanuk, I. Ueki, Dealing with methionine/homocysteine sulfur: cysteine metabolism to taurine and inorganic sulfur. J. Inherit. Metab. Dis. 34, 17-32 (2011).

60. N. Barghi, et al., Genetic redundancy fuels polygenic adaptation in Drosophila. PLoS Biol. 17, e3000128 (2019). 
61. N. Barghi, J. Hermisson, C. Schlötterer, Polygenic adaptation: a unifying framework to understand positive selection. Nat. Rev. Genet. 21, 769-781 (2020).

62. C. C. Steiner, H. Römpler, L. M. Boettger, T. Schöneberg, H. E. Hoekstra, The genetic basis of phenotypic convergence in beach mice: similar pigment patterns but different genes. Mol. Biol. Evol. 26, 35-45 (2009).

63. S. Yeaman, Local Adaptation by Alleles of Small Effect. Am. Nat. 186 Suppl 1, S74-89 (2015).

64. Y. B. Simons, K. Bullaughey, R. R. Hudson, G. Sella, A population genetic interpretation of GWAS findings for human quantitative traits. PLoS Biol. 16, e2002985 (2018).

65. A. F. Kautt, et al., Contrasting signatures of genomic divergence during sympatric speciation. Nature 588, 106-111 (2020).

66. S. M. Flaxman, A. C. Wacholder, J. L. Feder, P. Nosil, Theoretical models of the influence of genomic architecture on the dynamics of speciation. Mol. Ecol. 23, 4074-4088 (2014).

67. S. Renaut, et al., Genomic islands of divergence are not affected by geography of speciation in sunflowers. Nat. Commun. 4, 1827 (2013).

68. F. Han, et al., Gene flow, ancient polymorphism, and ecological adaptation shape the genomic landscape of divergence among Darwin's finches. Genome Res. 27, 1004-1015 (2017).

69. J. L. Feder, et al., Genome-wide congealing and rapid transitions across the speciation continuum during speciation with gene flow. J. Hered. 105 Suppl 1, 810-820 (2014).

70. C. R. Feldman, E. D. Brodie Jr., E. D. Brodie III, M. E. Pfrender, Constraint shapes convergence in tetrodotoxin-resistant sodium channels of snakes. Proc. Natl. Acad. Sci. U. S. A. 109, 4556-4561 (2012).

71. J. Parker, et al., Genome-wide signatures of convergent evolution in echolocating mammals. Nature 502, 228-231 (2013).

72. M. Schartl, et al., The genome of the platyfish, Xiphophorus maculatus, provides insights into evolutionary adaptation and several complex traits. Nat. Genet. 45, 567-572 (2013).

73. A. McKenna, et al., The Genome Analysis Toolkit: a MapReduce framework for analyzing nextgeneration DNA sequencing data. Genome Res. 20, 1297-1303 (2010).

74. R. N. Gutenkunst, R. D. Hernandez, S. H. Williamson, C. Bustamante, Inferring the joint demographic history of multiple populations from multidimensional SNP frequency data. PLoS Genet. 5, e1000695 (2009). 
75. M. Tobler, R. Riesch, C. M. Tobler, T. Schulz-Mirbach, M. Plath, Natural and sexual selection against immigrants maintains differentiation among micro-allopatric populations. J. Evol. Biol. 22, 2298-2304 (2009).

76. M. L. Zelditch, D. L. Swiderski, H. David Sheets, Geometric Morphometrics for Biologists: A Primer (Academic Press, 2012).

77. Adams, D. C., M. L. Collyer, A. Kaliontzopoulou, and E.K. Balken, Geomorph: Software for geometric morphometric analyses (2021).

78. T. Themeau, A package for survival analysis in $\mathrm{R}$ (2021).

79. M. Pertea, et al., StringTie enables improved reconstruction of a transcriptome from RNA-seq reads. Nat. Biotechnol. 33, 290-295 (2015).

80. M. Pertea, D. Kim, G. M. Pertea, J. T. Leek, S. L. Salzberg, Transcript-level expression analysis of RNA-seq experiments with HISAT, StringTie and Ballgown. Nat. Protoc. 11, 1650-1667 (2016).

81. Robinson, D. J. McCarthy, G. K. Smyth, edgeR: a Bioconductor package for differential expression analysis of digital gene expression data. Bioinformatics 26, 139-140 (2010).

82. Gene Ontology Consortium, The Gene Ontology (GO) database and informatics resource. Nucleic Acids Res. 32, D258-D261 (2004).

83. N. Dierckxsens, P. Mardulyn, G. Smits, NOVOPlasty: de novo assembly of organelle genomes from whole genome data. Nucleic Acids Res. 45, e18 (2017).

84. W. Iwasaki, et al., MitoFish and MitoAnnotator: a mitochondrial genome database of fish with an accurate and automatic annotation pipeline. Mol. Biol. Evol. 30, 2531-2540 (2013).

85. J. W. Leigh, D. Bryant, Popart : Full-feature software for haplotype network construction. Methods Ecol. Evol. 6, 1110-1116 (2015).

86. Z. Yang, PAML 4: Phylogenetic analysis by maximum likelihood. Mol. Biol. Evol. 24, 1586-1591 (2007). 

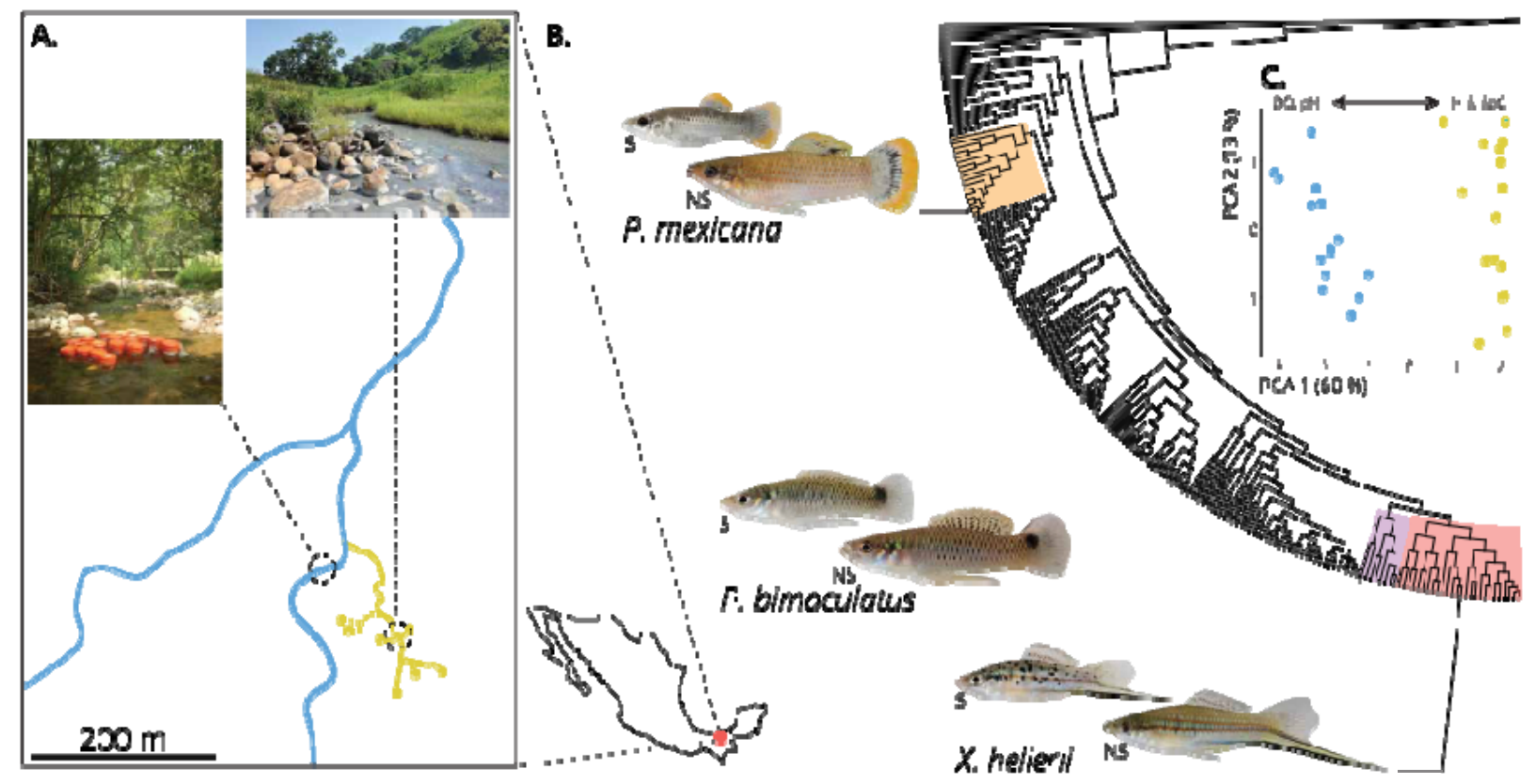

Figure 1. Sympatric populations of three highly divergent poeciliid lineages co-occur in a sulfide spring and adjacent nonsulfidic stream in southern Mexico. A. Map of the study site, where yellow points indicate the $\mathrm{H}_{2} \mathrm{~S}$ spring heads of the La Gloria spring system, while yellow lines indicate sulfide-rich stream segments. Blue lines indicate the adjacent nonsulfidic stream system, Arroyo Caracol. Inset photos depict representative habitats within the two streams (top: La Gloria, sulfidic; left: Arroyo Caracol, nonsulfidic). B. Phylogeny of the family Poeciliidae highlighting the three focal genera: Poecilia (orange), Pseudoxiphophorus (purple), and Xiphophorus (red). Each species' position within its genus is denoted by the extended branch leading to representative photographs of a sulfidic (S; top) and nonsulfidic (NS; bottom) male of each species. C. Nonsulfidic and sulfidic habitats differed significantly in water chemistry. Principal component analysis indicated that sites within the La Gloria spring complex (yellow) and Arroyo Caracol (blue) separated along the primary axis of variation, with nonsulfidic sites exhibiting higher dissolved oxygen concentrations (DO) and $\mathrm{pH}$ and sulfidic sites exhibiting higher $\mathrm{H}_{2} \mathrm{~S}$ concentrations and specific conductivities ( $\mathrm{SpC}$ ). 

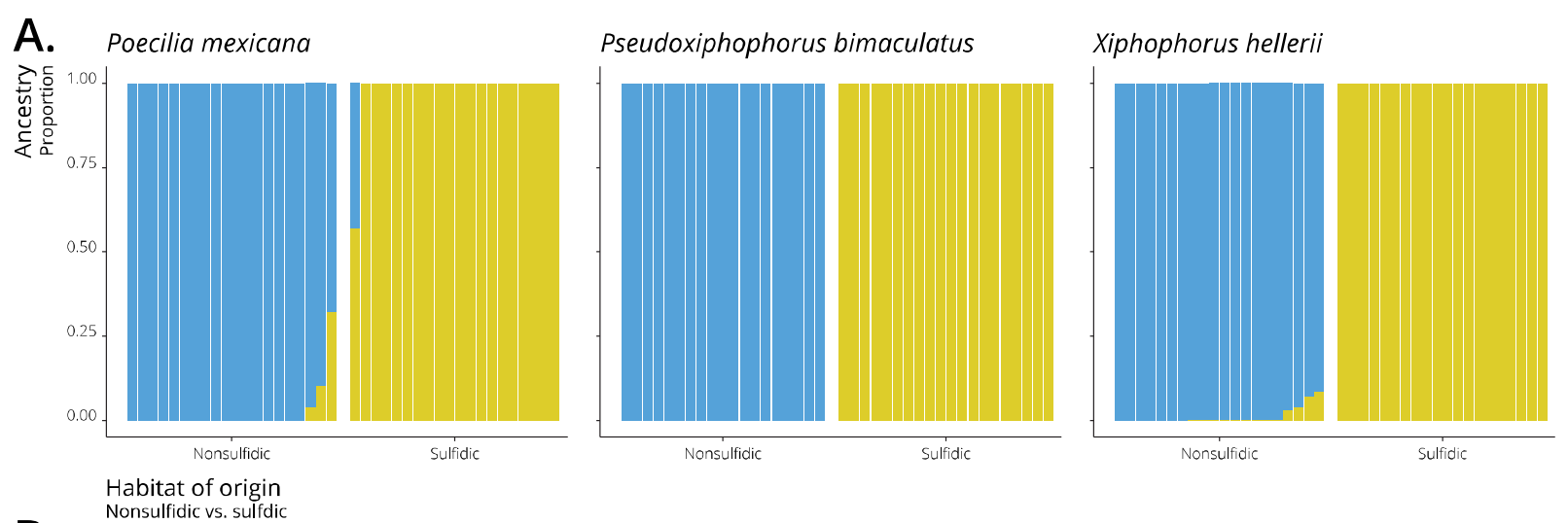

B.
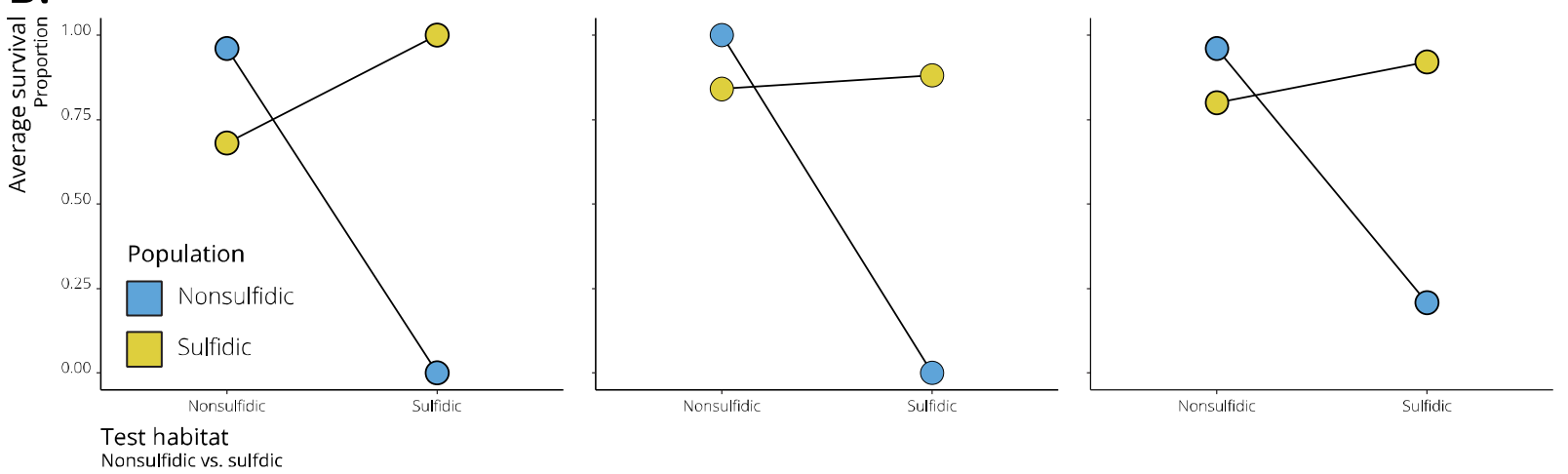

Figure 2. Nonsulfidic and sulfidic populations of three sympatric species exhibit high levels of reproductive isolation mediated by strong natural selection against migrants. A. Admixture analyses revealed substantial population genetic differentiation in each population pair. Individuals are grouped by sampling site, corresponding with the detected genetic population clusters $(K=2)$ shown in blue (nonsulfidic) and yellow (sulfidic). B. Translocation experiments revealed strong selection against migrants, especially those from nonsulfidic to sulfidic habitats (Table S1). Fish were transferred between sites (test habitat) to estimate survival of migrants between habitats, with individuals collected from the nonsulfidic population in blue and from the sulfidic population in yellow. 


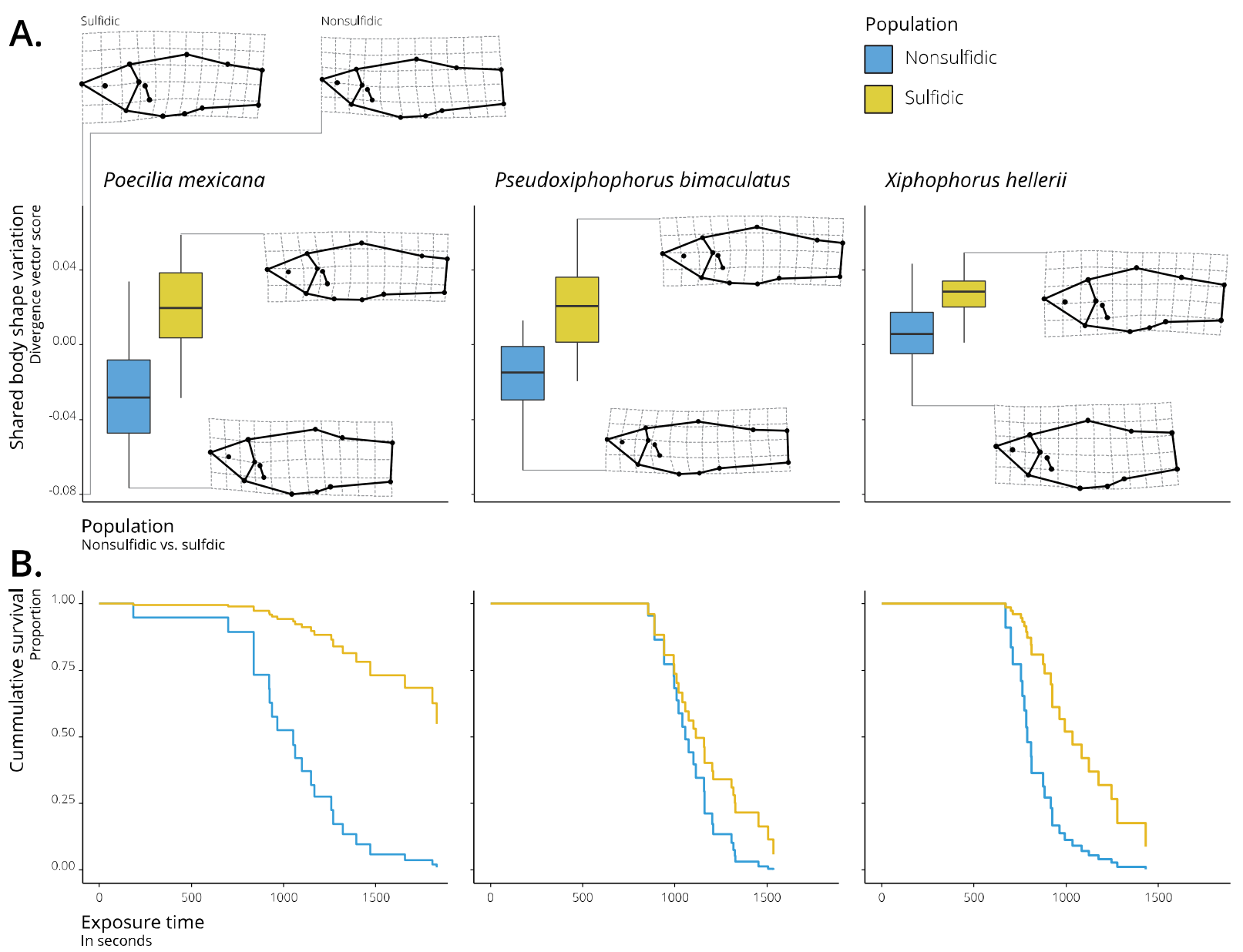

Figure 3. Phenotypic convergence in morphological and physiological traits of sympatric sulfidic populations. A. Convergent morphological evolution among species between habitat types. Body shape differentiation along a shared axis of divergence (habitat type) is depicted by the transformation grids on the top. Significant differences in body shape between populations of each species is depicted in insets (Table S2). B. Convergence in $\mathrm{H}_{2} \mathrm{~S}$ tolerance among all populations from the sulfidic habitat. Cumulative survival in acute $\mathrm{H}_{2} \mathrm{~S}$ toxicity trials revealed significant differences between populations, which were most pronounced in P. mexicana (Table S3). 

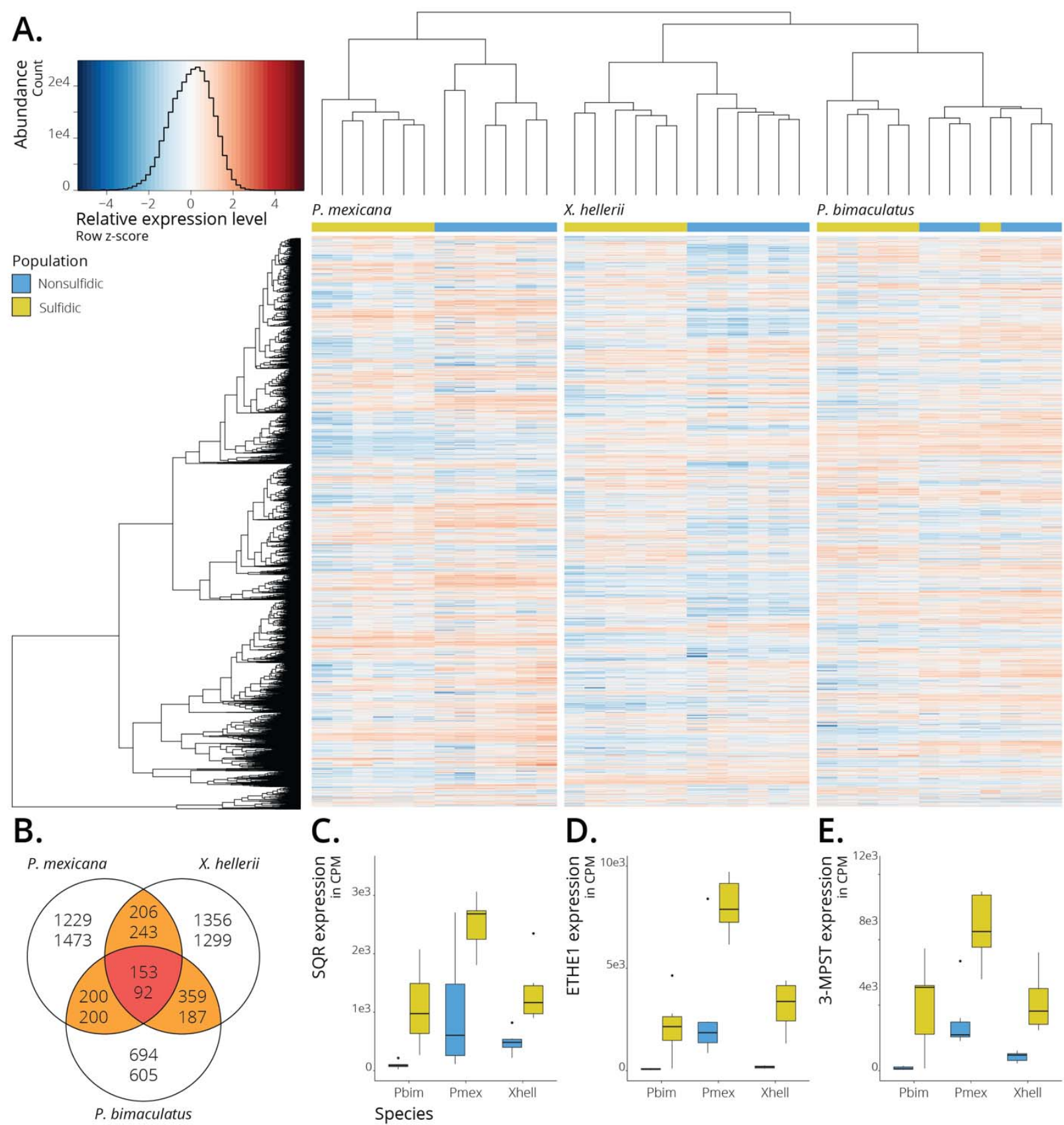
X. hellerii P. bimaculatus
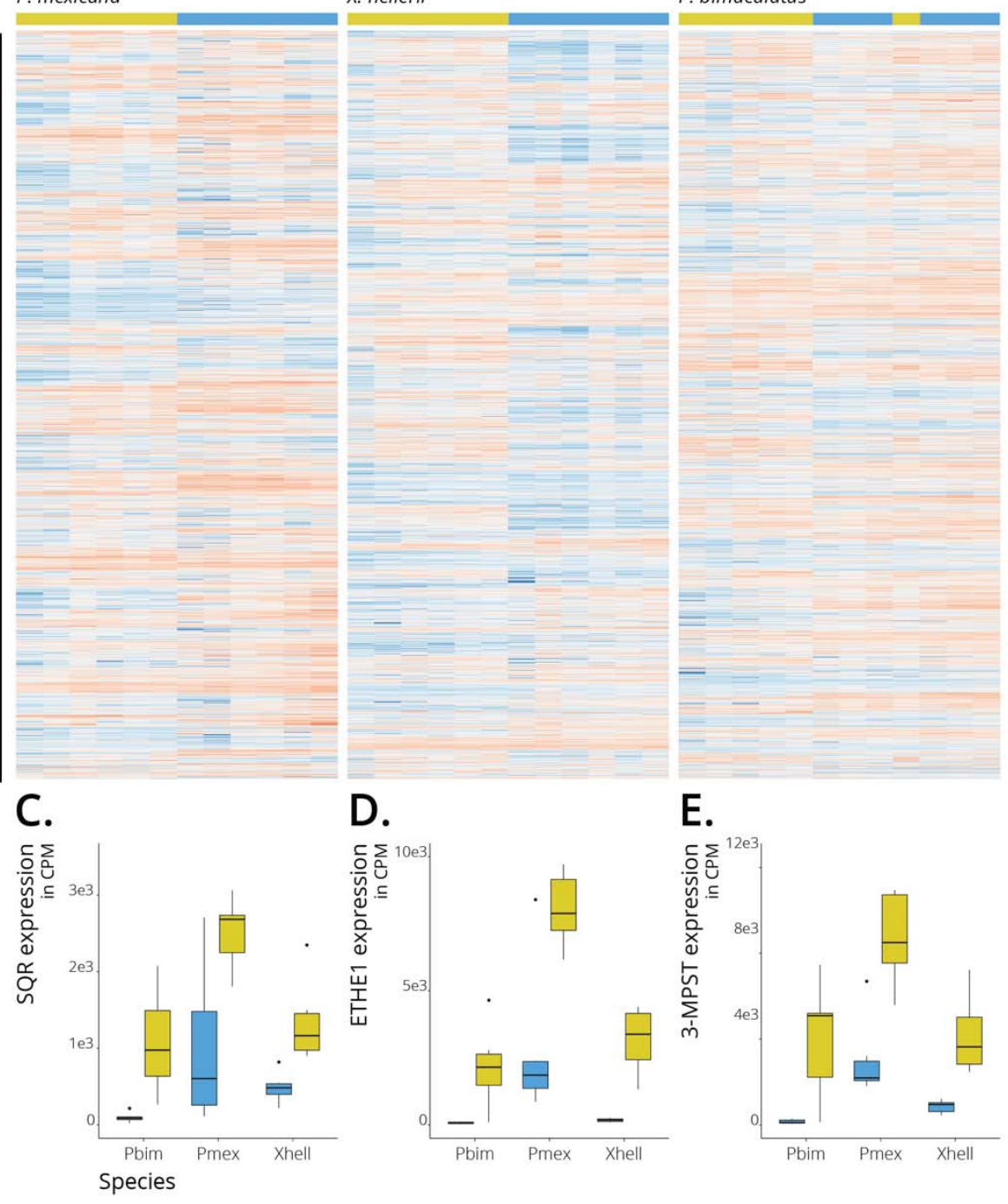

Figure 4. Convergent shifts in gene expression underlie local adaptation. A. Hierarchical clustering analysis indicates that gene expression profiles cluster by habitat type within each species. Each row represents expression data for one gene, and each column corresponds to an individual. Genes with higher relative expression are indicated in red, genes with lower relative expression in blue. B. Venn diagram showing the number of differentially expressed genes between the sulfidic and nonsulfidic populations of each species and those shared among species. Top numbers represent upregulated genes, bottom numbers downregulated genes. C-E. Sulfidic populations consistently exhibit higher expression of key $\mathrm{H}_{2} \mathrm{~S}$ detoxification genes (SQR: sulfide:quinone oxidoreductase; ETHE1: persulfide dioxygenase; 3-MPST: 3-mercaptopyruvate sulfurtransferase) than nonsulfidic populations of the same species. 
A.
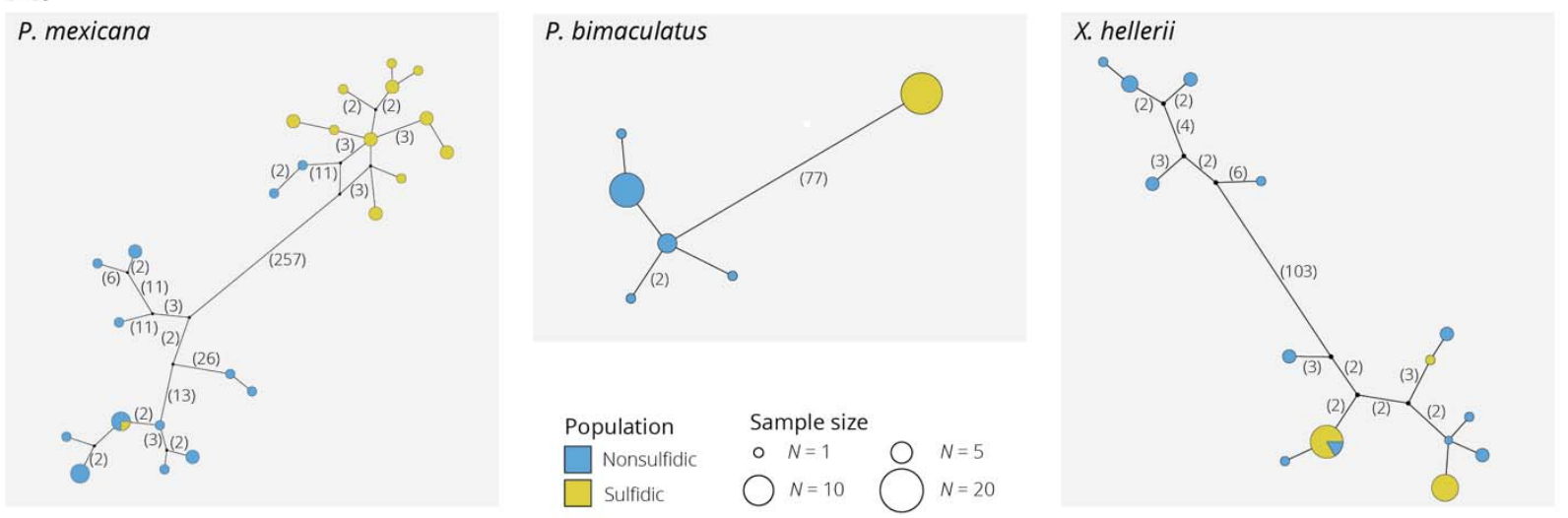

B.
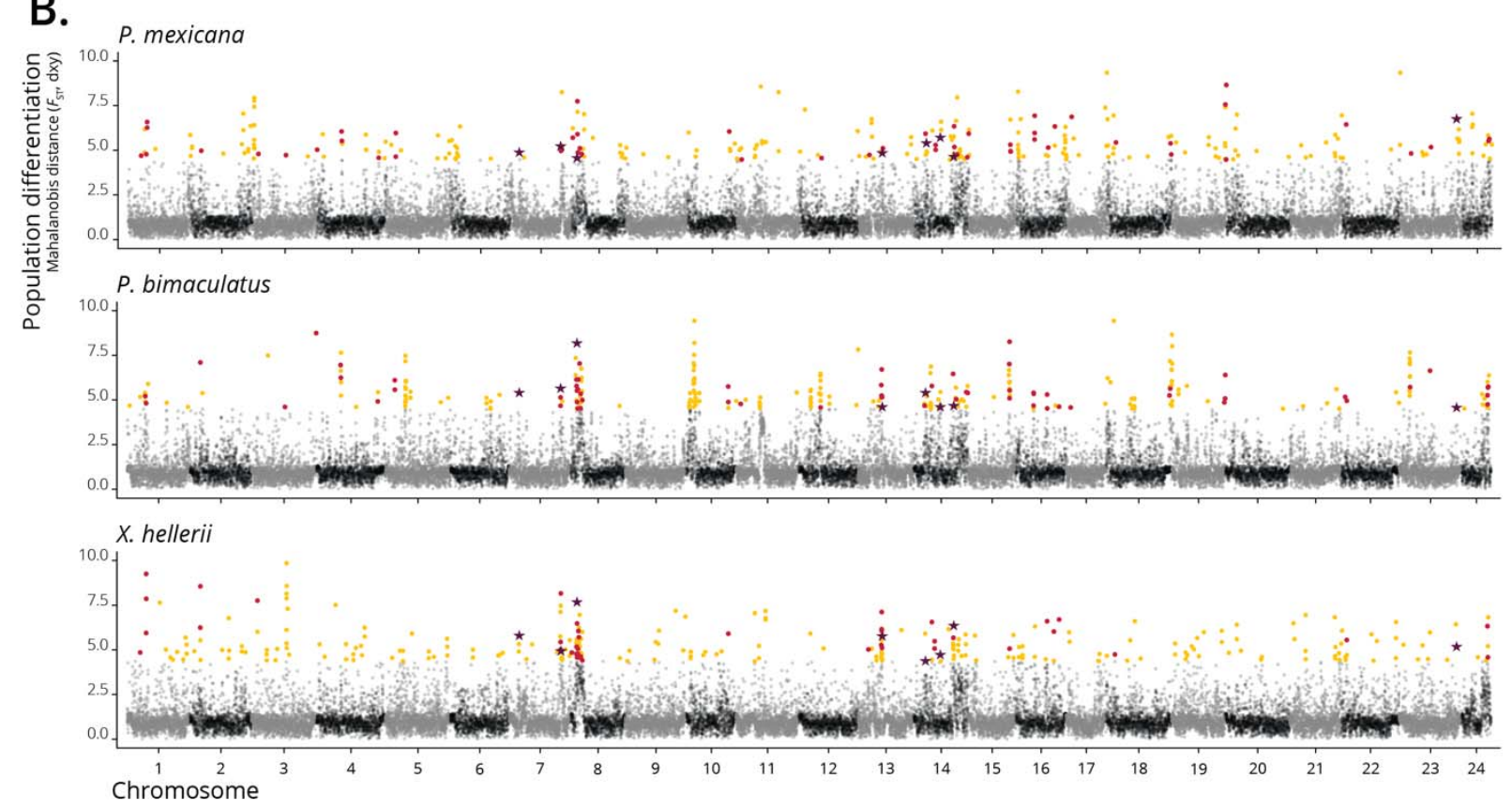

Figure 5. Convergent patterns of genomic adaptation are limited to the mitochondrial genome and few nuclear loci. A. Haplotype networks based on concatenated mitochondrial genes indicate high levels of mitochondrial divergence between nonsulfidic (blue) and sulfidic (yellow) populations, with asymmetrical introgression of sulfidic mitochondria into nonsulfidic individuals. The relative size of each circle indicates the number of individuals with a particular mitochondrial haplotype, while numbers in parentheses represent the total number of nucleotide differences between haplotypes. B. Mahalanobis distance $\left(-\log _{10} P\right.$-values of $F_{\mathrm{ST}}$ and $\left.d_{\mathrm{XY}}\right)$ of non-overlapping 25 -kb windows across the genome as a measurement of genomic divergence driven by natural selection. Outlier windows (top $1 \%$ ) unique to each population pair are shown in yellow, while those shared between two population pairs are in red. The eight outlier windows shared among all three population pairs are denoted with burgundy stars. 